\title{
METHOD OF ACOUSTIC CALCULATION OF TRAFFIC NOISE BARRIERS
}

\author{
Tikhon Dudiev, Vladimir Tupov* \\ B auman M oscow State Technical University, 105005, M oscow, Russia
}

\begin{abstract}
Installation of noise barriers is an efficient and relatively cheap way of protecting residential areas from traffic noise. This paper proposes a comprehensive method of calculation of noise barriers. The method allows determining the optimal sizes of the barrier meeting acoustic and cost requirements. The calculation method takes into account the noise coming from the both parts of the traffic flow shielded and not shielded by the barrier, as well as sound reflected from the road surface, the opposite barrier, if present, and both barriers.
\end{abstract}

\section{Introduction}

High noise pollution of territories close to traffic flows is a serious environmental problem. Despite the reduction of car noise by creating low-noise engines, more efficient mufflers [15], etc., the desired effect is not achieved, and the problem of noise reduction remains relevant. There are many ways of protecting building close to traffic flow from noise. For example, there is personal protective equipment (ear plugs, headphones) and collective protective equipment, including soundproof windows and the use of noise-absorbing materials. However, noise barriers (NB) is the most effective and cost-efficient way of protecting buildings from noise. This paper proposes a comprehensive method of calculating the optimal parameters of NBs. This method ensures the required NB efficiency at the lowest cost.

\section{Analysis of works on the research topic and setting a research task}

Literature review of methods of design and simulation of NBs revealed that many researchers neglect a number of factors influencing the NB efficiency or focus solely on individual design aspects without taking into account, for example, the noise coming from the traffic flow not covered by the NB. Researches have to make assumptions to simplify the design problem. For example, some studies consider infinitely long screens [6, 7], others consider only point sources $[8,9]$, while some studies offer calculation methods that ensure sufficient accuracy in a limited frequency range [10]. There are works that take into account the limited barrier length and the loss of acoustic energy during the propagation of sound from traffic

* Corresponding author: vvtupov@mail.ru 
flow to the estimated point (EP) [11,12]. Paper [13] proposed a method of determining the acoustic efficiency of a noise barrier and its dimensions and proposed recommendations on improving the NB construction.

Thus, there is a need to develop a comprehensive methodology that will meet all the requirements for the construction of noise screens and the calculation of its effectiveness. The proposed method takes into account the influence of the wind and atmosphere turbulence on sound propagation, interaction of acoustic waves with the surfaces of the territory and wood lines. Also, the simulation takes into account the noise coming to the protected territory from part of the traffic flow closed by the barrier and the parts not closed by it, as well as sound reflected from the road surface, the opposite barrier (if present) and both barriers.

\section{Calculation of direct and reflected sound levels from the traffic flow in the estimated point with NB}

To calculate the levels of direct and reflected sound from the traffic flow in the estimated point with NB, we use the results of studies presented in [14].

First, we calculate the level of direct sound that is created by the traffic flow at the estimated point:

$$
L_{\text {dir }}=L_{n}-\Delta L_{n b}-\Sigma \Delta L
$$

where $L_{n}$ is the noise characteristic of the traffic flow; $\Delta L_{n b}$ is the noise reduction by NB; $\Sigma \Delta L$ is the sum of all sound energy losses on the sound propagation path from is source to the estimated point.

During propagation from the source to the estimated point, the noise is reduced due to the following factors: losses from the surface of the protected territory, losses in the air, losses due to wind, atmosphere turbulence, influence of wood lines, losses due to the limited barrier length and divergence of acoustic waves. The influence on the noise level at the estimated point of reflected sound can be taken into account by introducing three imaginary sources each of which creates a sound level in the estimated point:

$$
L_{r e f l, i}=L_{\mathrm{n}}+\Delta L_{R, i}-\Sigma \Delta L_{i}-\Delta L_{\mathrm{NB}, i}
$$

where $\Delta L_{R, i}=10 \lg \left(R_{i}\right)$ is the noise reduction of the $i$-th imaginary source when the traffic noise is reflected from a reflecting object; $R_{i}$ is the sound reflection coefficient (for reinforced concrete barriers $R_{i} \approx 0,97$ [15]; for asphalt and asphalt-concrete road surfaces $R_{i} \approx 0.955$ [16]); $\Sigma \Delta L_{i}$ is the sum of acoustic energy losses during propagation of sound from the $i$-th imaginary source to the estimated point; $\Delta L_{\mathrm{NB}, i}$ is the noise barrier reduction of sound from the i-th imaginary sources (equations for calculating $\Delta L_{\mathrm{NB}, i}$ are shown below).

As a first approximation, the sum of reflected sound losses $\left(\Sigma \Delta L_{\mathrm{i}}\right)$ can be assumed equal, it is denoted as $\Sigma \Delta L_{\text {refl }}$. The total sound level in the estimated point created by three considered imaginary sources is given by:

$$
L_{r e f l, \Sigma}=L_{\mathrm{n}}+10 \lg \left(\Sigma_{\mathrm{i}=1}^{3} 10^{0.1\left(\Delta L_{\mathrm{R}, i}-\Delta L_{\mathrm{NB}, i}\right)}\right)-\Sigma \Delta L_{r e f l} .
$$

The sound level in the estimated point created by the direct and reflected sound:

$$
L_{\Sigma}=10 \log \left(10^{0,1 L_{d i r}}+10^{0,1 L_{r e f l, \Sigma}}\right) .
$$


The increase of the sound level in the estimated point due to reflected sound is given by:

$$
\Delta L=L_{\Sigma}-L_{d i r}
$$

\section{Calculation of reduction by the noise barrier of the sound reflected from the road surface}

To calculate reduction by the noise barrier of the sound reflected from the road surface, we use the results of study presented in [14]. Calculation diagram for determining the difference of the path lengths of the sound ray $\delta$ during reflection from the road surface is shown in Fig. 1.

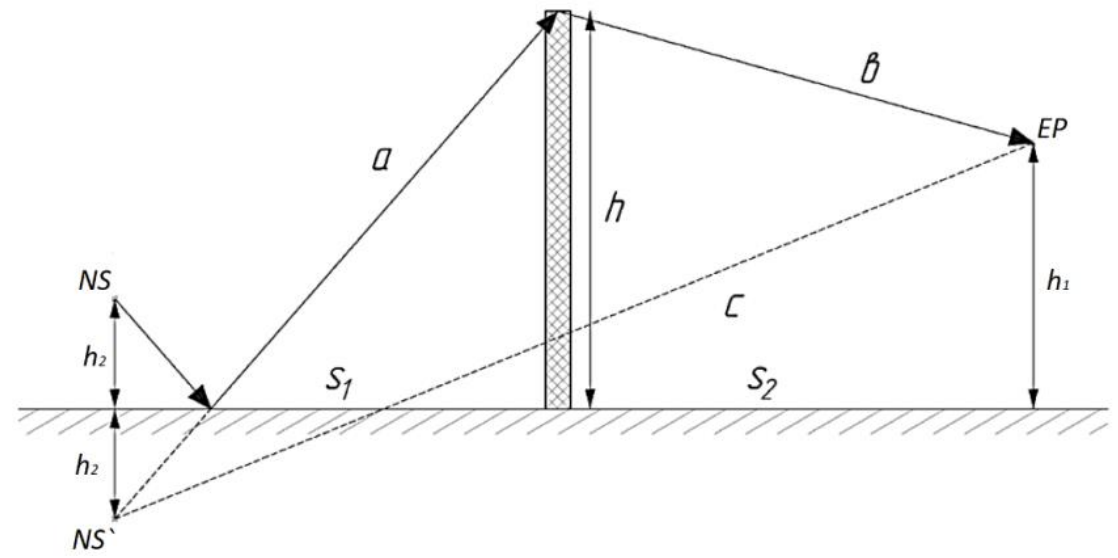

Fig.1. Calculation diagram for determining difference of the path lengths of the sound ray $\delta$ during reflection of sound from the road surface

Reduction of the sound level reflected from the road surface by the noise barrier [17] is given by:

$$
\Delta L_{N B, i}=18,2+7,8 \lg (\delta+0,02), \mathrm{dBA},
$$

where $\delta$ is the difference of path lengths of the sound rays, m:

$$
\delta=a+b-c
$$

$a$ - the distance between the acoustic center of the imaginary noise source and the upper edge of the barrier, m:

$$
a=\sqrt{s_{1}^{2}+\left(h+h_{2}\right)^{2}}
$$

$b$ - the distance from the highest edge of the barrier to the estimated point, $\mathrm{m}$ :

$$
b=\sqrt{s_{2}^{2}+\left(h-h_{1}\right)^{2}}
$$

$c$ - the shortest distance from the acoustic center of the imaginary source to the estimated point, m: 


$$
c=\sqrt{\left(s_{1}+s_{2}\right)^{2}+\left(h_{1}+h_{2}\right)^{2}}
$$

$h$ - noise barrier height, m;

$h_{1}$ - height of the estimated point above the surface of the territory, m;

$h_{2}$ - height of the NS acoustic center above the road surface, m;

$s_{1}$ and $s_{2}$ is the distance from the $N B$ to axis of the far lane and the estimated point, accordingly, $\mathrm{m}$.

\section{Calculation of reduction by the noise barrier of the sound reflected from the screen located on the opposite side of the road}

To calculate of reduction by the noise barrier of the sound reflected from the screen located on the opposite side of the road, we use the result of [14]. The calculation diagram is shown in Fig. 2.

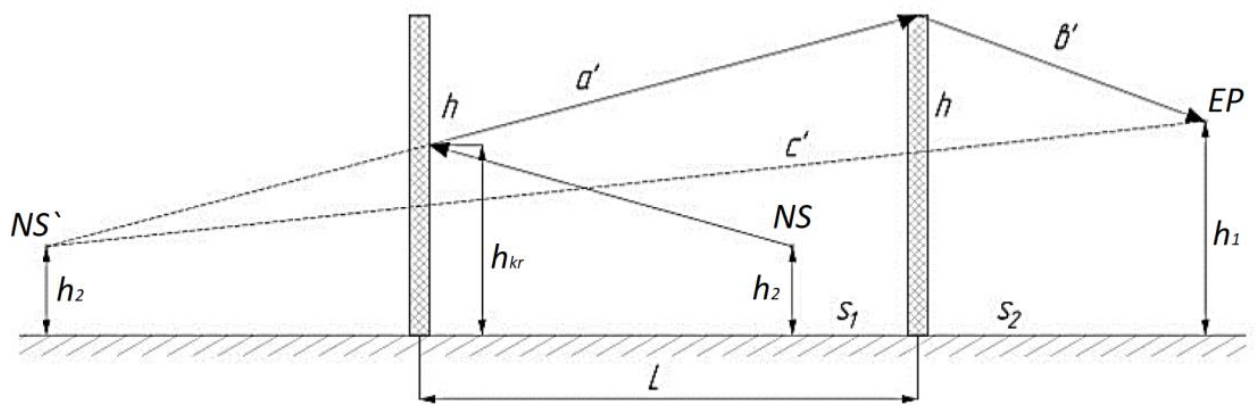

Fig.2. Calculation diagram for determining the parameter $\delta$ for the sound reflected from the opposite barrier

Similarly, to (6), we determine of reduction by the noise barrier of the sound reflected from the parallel noise barrier on the opposite side of the road. The difference of path lengths of the sound rays $\delta$ is given by:

$$
\delta=a^{\prime}+b^{\prime}-c^{\prime}
$$

$a^{\prime}$ - the distance between the acoustic center of the imaginary noise source and the upper edge of the barrier, $\mathrm{m}$ :

$$
a^{\prime}=\sqrt{\left(2 L-s_{1}\right)^{2}+\left(h-h_{2}\right)^{2}} ;
$$

$b^{\prime}$ - the distance from the upper edge of the barrier to the estimated point, m:

$$
b^{\prime}=\sqrt{s_{2}^{2}+\left(h-h_{1}\right)^{2}} ;
$$

$c^{\prime}-$ the shortest distance from the acoustic center of the imaginary noise source to the estimated point, $\mathrm{m}$ :

$$
c^{\prime}=\sqrt{\left(2 L-s_{1}+s_{2}\right)^{2}+\left(h_{1}-h_{2}\right)^{2}}
$$

where $s_{1}$ and $s_{2}$ - are the distances from the noise barrier to the axis of the closest traffic lane and the estimated point, accordingly, $\mathrm{m}$; 
$L$ the distance between parallel noise barriers installed on the opposite sides of the carriageway of the road, $\mathrm{m}$.

\section{Calculation of reduction by the noise barrier of the sound reflected from the NB and the barrier located on the other side of the road}

For calculation of reduction by the noise barrier of the sound reflected from the noise barrier and the barrier located on the other side of the road, we use equations presented in [14]. The calculation diagram is shown in Fig. 3.

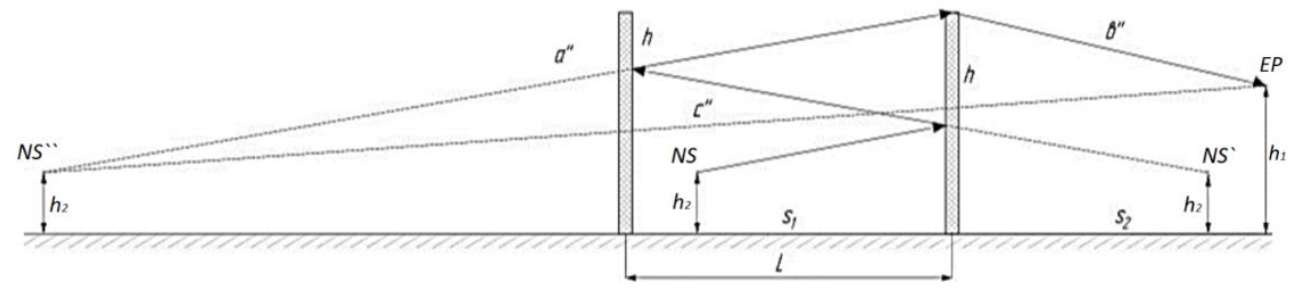

Fig.3. Calculation diagram for determining the difference of the path length of the sound ray $\delta$ for the double reflection from both noise barriers

Similar to (6), we calculate the noise barrier reduction of noise reflected from both parallel noise barriers. The difference of the path lengths of the sound rays $\delta$ is given by:

$$
\delta=a^{\prime \prime}+b^{\prime \prime}-c^{\prime \prime}
$$

$a^{\prime \prime}$ - the distance between the acoustic center of the imaginary sound source and upper edge of the barrier, $\mathrm{m}$ :

$$
a^{\prime \prime}=\sqrt{\left(2 L+S_{1}\right)^{2}+\left(h-h_{2}\right)^{2}}
$$

$b^{\prime \prime}$ - the distance from the upper edge of the barrier to the estimated point, $\mathrm{m}$ :

$$
b^{\prime \prime}=\sqrt{s_{2}^{2}+\left(h-h_{1}\right)^{2}} \text {; }
$$

$c^{\prime \prime}-$ is the closest distance from the acoustic center of the imaginary source to the estimated point, m:

$$
c^{\prime \prime}=\sqrt{\left(2 L+s_{1}+s_{2}\right)^{2}+\left(h_{1}-h_{2}\right)^{2}} ;
$$

$s_{1}$ and $s_{2}-$ is the distance from the NB to axis of the far lane and the estimated point, accordingly, $\mathrm{m}$.

\section{Calculation of the levels of noise on the protected territory generated by unshielded parts of the traffic flow}

To calculate the levels of noise on the protected territory generated by the parts of the unshielded parts of the traffic flow, we use the results presented in [18]. The calculation diagram is shown in Fig. 4. 


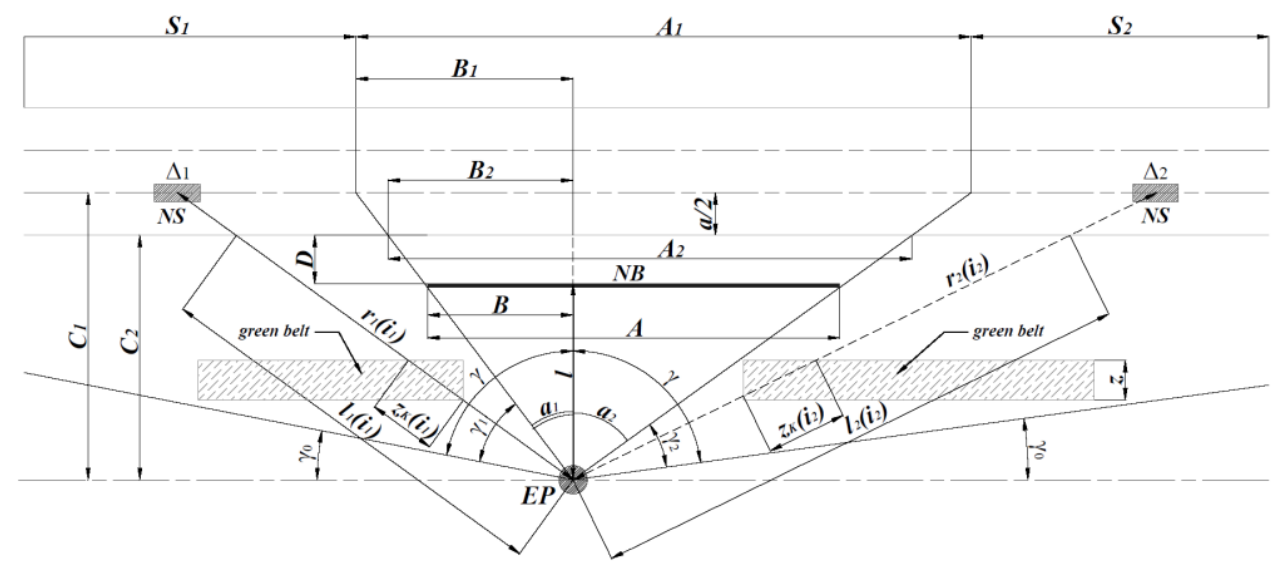

Fig. 4. Calculation diagram for evaluating the noise at the estimated point in the territory protected by the NB, created by sections of the traffic flow that are not covered by the NB

As a first approximation, the noise characteristic of the traffic flow [19] is given by an empirical equation:

$$
\begin{aligned}
L_{A}^{e q}= & 10 \lg N+13.3 \lg V+8.4 \lg r+4(s-0.13)^{-1}-0.0079 s^{2}+ \\
& +0.5 q \lg (0.5 r+2.8)+P+8,5, \mathrm{dBA}
\end{aligned}
$$

where $N$ - traffic intensity (number of traffic units passing in both directions per one hour), $1 / \mathrm{h}$;

$V$ - weighted average car traffic speed, $\mathrm{km} / \mathrm{h}$;

$r$ - the share of freight vehicles and public transport vehicles in the traffic flow, \%;

$s$ - number of traffic lanes;

$q$ - longitudinal slope of the road, \%;

$P$ - road surface type ( $P=0$ for asphalt concrete, $P=3 \mathrm{dBA}$ for cement concrete).

We determine the angles of visibility of parts of the unshielded traffic flow from the estimated point:

- to the left of the barrier:

$$
\gamma_{1}=\gamma-\alpha_{1}, \operatorname{rad}
$$

- to the right of the barrier:

$$
\gamma_{2}=\gamma-\alpha_{2}, \operatorname{rad}
$$

where

$$
\gamma=\pi / 2-\gamma_{0}
$$

(see Fig. 4) here $\gamma_{0}$ - the angle for which the vehicles almost do not contribute to the total noise level in the estimated point, this value is set to $3^{\circ}$;

$\alpha_{1}$ and $\alpha_{2}$ - visibility angles of the left- and right-hand sides of the noise barrier from the estimated point.

Length of the unshielded traffic flow on the lane closest to the estimated point to the left and right of the noise barrier, Fig. 4, is given by:

- left: 


$$
\mathrm{S}_{1}=\mathrm{c}_{1} \operatorname{tg} \gamma-\mathrm{B}_{1}, \mathrm{~m}
$$

- right:

$$
\mathrm{S}_{2}=\mathrm{c}_{1} \operatorname{tg} \gamma-\mathrm{A}_{1}+\mathrm{B}_{1}, \mathrm{~m}, \quad \text { (24) }
$$

where $c_{1}=l+D+a / 2$ - the distance from the estimated point to the axis of the closest traffic lane, $\mathrm{m}$;

1 and $\mathrm{D}-$ the distance from the $N B$ to the estimated point and the shoulder of the road, $\mathrm{m}$; a - width of one traffic lane, $\mathrm{m}$;

$\mathrm{A}_{1}$ - the length of the equivalent line of the NS limited by two rays passing through side edges of the barrier from the estimated point, Fig. 4:

$$
\mathrm{A}_{1}=\mathrm{Ac}_{1} / \mathrm{l}, \mathrm{m}
$$

$B_{1}$ - the section of length $A_{1}$ limited by the line normal to the traffic trajectory and the left ray limiting the line section $A_{1}$,

$$
\mathrm{B}_{1}=\mathrm{Bc}_{1} / \mathrm{l}, \mathrm{m}
$$

where A - noise barrier length, $\mathrm{m}$;

$\mathrm{B}-$ length of the part of the barrier visible at the angle of $\alpha 1$ from the estimated point, Fig. 4, m.

Let us break down the open parts of the traffic flow represented as line sections with length $S_{1}$ and $S_{2}$ in the model into finite elements with the length of $\Delta$ [20]. The element length is a function of traffic intensity $N$ and speed $V$ :

$$
\Delta=1000 \mathrm{~V} / \mathrm{N}, \mathrm{m} \text {. }
$$

Let us determine the initial number of elements on these sections: - left:

$$
\mathrm{n}_{1}=\mathrm{S}_{1} / \Delta
$$

- right:

$$
\mathrm{n}_{2}=\mathrm{S}_{2} / \Delta
$$

Let us round up the values $n_{1}$ and $n_{2}$ to the integer values of $m_{1}$ and $m_{2}$ and recalculate the element lengths $\Delta_{1}, \Delta_{2}$ for sections $\mathrm{S}_{1}, \mathrm{~S}_{2}$ :

- left:

- right:

$$
\Delta_{1}=\mathrm{S}_{1} / \mathrm{m}_{1}, \mathrm{~m}
$$

$$
\Delta_{2}=\mathrm{S}_{2} / \mathrm{m}_{2}, \mathrm{~m}
$$

The finite elements are numbered as follows:

- left: $i_{1}=1,2 \ldots \mathrm{m}_{1}$;

- right: $i_{2}=1,2 \ldots \mathrm{m}_{2}$. 
Let us calculate the distance from the estimated point to the $i$-th element of the traffic flow model:

- left:

$$
r_{1}\left(i_{1}\right)=\sqrt{\left[B_{1}+\Delta_{1}\left(2 i_{1}-1\right) / 2\right]^{2}+R^{2}}, \mathrm{~m}
$$

- right:

$$
r_{2}\left(i_{2}\right)=\sqrt{\left[A_{1}-B_{1}+\Delta_{2}\left(2 i_{2}-1\right) / 2\right]^{2}+R^{2}}, \mathrm{~m}, \quad \text { (34) }
$$

where $R=\sqrt{\left(h_{1}-h_{2}\right)^{2}+c_{1}^{2}}-$ the distance from the estimated point to the acoustic center of a vehicles measured in direction normal to the traffic flow, (35)

$h_{1}$ and $h_{2}$ - the height of the estimated point and the height of the acoustic center of the noise source above the surface of the territory, $\mathrm{m}$.

Let us determine the reduction of the sound level due to its divergence from the $i$-th elements of the traffic flow model:

$$
\Delta L_{R, K}\left(i_{K}\right)=20 \lg \left[\mathrm{r}_{K}\left(i_{\mathrm{K}}\right)\right], \mathrm{dBA},
$$

where $K$ - the index for writing equations in the general form, while $\mathrm{K}=1$ is for the parts of the traffic flow to the left of the NB and $K=2$ is for the traffic flow parts to the right of the NB.

We determine the noise reduction in air during propagation of acoustic waves from $\mathrm{i}$-th elements to the estimated point:

$$
\Delta L_{a i r, K}\left(i_{K}\right)=0,005 \sqrt{\left(h_{1}-h_{2}\right)^{2}+r_{K}\left(i_{K}\right)^{2}}, \mathrm{dBA} .
$$

We determine the reduction of sound due to wind and atmosphere turbulence:

$$
\Delta L_{\mathrm{B}, \mathrm{T}, \mathrm{K}}\left(i_{K}\right)=3\left[1,6+10^{5} / r_{K}\left(i_{K}\right)^{2}\right]^{-1}, \mathrm{dBA} .
$$

We determine the losses from the interaction of the acoustic waves and the territory surface using equations derived in [19]:

- for acoustically rigid surface: $\Delta L_{s u r f, K}\left(i_{K}\right)=0$;

- for acoustically soft surface:

$$
\left\{\begin{array}{l}
\Delta L_{\text {surf }, K}\left(i_{K}\right)=E\left(i_{K}\right)-9,775 \text { if } l_{K}\left(i_{K}\right) \leq 100 \mathrm{~m} \\
\Delta L_{\text {surf }, K}\left(i_{K}\right)=E\left(i_{K}\right)-0,15 \ln \left[l_{K}\left(i_{K}\right)-100\right]-9,775 \text { if } 100 m<l_{K}\left(i_{K}\right)<170 \mathrm{~m} ; \\
\Delta L_{\text {surf }, K}\left(i_{K}\right)=E\left(i_{K}\right)-0,1\left\{\ln \left[l_{K}\left(i_{K}\right)-170\right]\right\}^{2} \text { if } l_{K}\left(i_{K}\right) \geq 170 \mathrm{~m}
\end{array}\right.
$$

where $E\left(i_{K}\right)=5 \ln \left[l_{K}\left(i_{K}\right) / h_{1}\right]-3,45 h_{2}$.

The distance travelled by the sound waves from the $i_{1}$-th element and the $i_{2}$-the element to the estimated point above the ground surface measured from the shoulder of the road is given by:

$$
l_{1}\left(i_{1}\right)=\sqrt{\left[B_{2}+\Delta_{1}^{\prime}\left(2 i_{1}-1\right) / 2\right]^{2}+c_{2}^{2}}, \mathrm{~m}
$$




$$
l_{2}\left(i_{2}\right)=\sqrt{\left[A_{2}-B_{2}+\Delta_{2}^{\prime}\left(2 i_{2}-1\right) / 2\right]^{2}+c_{2}^{2}}, \mathrm{~m},
$$

where $\quad \Delta_{1}=\Delta_{1} c_{2} / c_{1}$ and $\Delta_{2}=\Delta_{2} c_{2} / c_{1}$ - lengths of the traffic flow elements relative to the closest shoulder of the road;

$c_{2}=l+D-$ the distance from the estimated point to the shoulder of the road, Fig. 4, m;

$A_{2}=A c_{2} / l$ and $B_{2}=B c_{2} / l$ - length of the noise barrier and the length of its part relative to the shoulder of the road closest to the estimated point.

We calculate the noise reduction by the green belt [13], if present, using relations:

$$
\begin{aligned}
& \Delta L_{\text {green }, 1}\left(i_{1}\right)=0,08 Z_{1}\left(i_{1}\right), \mathrm{dBA} \\
& \Delta L_{\text {green }, 2}\left(i_{2}\right)=0,08 Z_{2}\left(i_{2}\right), \mathrm{dBA} \\
& Z_{1}\left(i_{1}\right)=z_{1} / \cos \left\{\operatorname{arctg}\left[\left(B_{1}+\Delta_{1}\left(2 i_{1}-1\right) / 2\right) / R\right]\right\}, \mathrm{m} ; \\
& Z_{2}\left(i_{2}\right)=z_{2} / \cos \left\{\operatorname{arctg}\left[\left(A_{1}-B_{1}+\Delta_{2}\left(2 i_{2}-1\right) / 2\right) / R\right]\right\}, \mathrm{m} ;
\end{aligned}
$$

where $10 \mathrm{~m} \leq z_{K} \leq 100 \mathrm{~m}-$ is the width of the green belt.

We calculate the sound level in the estimated point generated by the $i$-th element of the model of the left- and right-hand sides of the unshielded traffic flow:

$$
\begin{gathered}
L_{A, i, K}\left(i_{K}\right)=L_{A}^{e q}+10 \lg \left(3,75 \Delta_{K}\right)-\Delta L_{R, K}\left(i_{K}\right)- \\
-\Delta L_{\text {air }, K}\left(i_{K}\right)-\Delta L_{B, T, K}\left(i_{K}\right)-\Delta L_{\text {surf }, K}\left(i_{K}\right)-\Delta L_{\text {green }, K}\left(i_{K}\right), \text { dBA. }
\end{gathered}
$$

We calculate the individual sound levels from the left- and right-hand unshielded parts of the traffic flow:

$$
\begin{aligned}
& L_{A, 1}=10 \lg \left[\sum_{i_{1}=1}^{m_{1}} 10^{0,1 L_{A, i, 1}\left(i_{1}\right)}\right], \mathrm{dBA} \\
& L_{A, 2}=10 \lg \left[\sum_{i_{2}=1}^{m_{2}} 10^{0,1 L_{A, i, 2}\left(i_{2}\right)}\right], \mathrm{dBA} .
\end{aligned}
$$

We calculate the total sound level in the estimated point from acoustic emission of both parts of the traffic flow unshielded by the noise barrier:

$$
L A 1=10 \lg \left[10^{0,1 L_{A, 1}}+10^{0,1 L_{A, 2}}\right], \mathrm{dBA} .
$$

\section{Calculation of the levels of sound in the estimated point with diffraction of sound on the top and side edges of the noise barrier}

To calculate the levels of sound in the estimated point with diffraction of sound on the top and side edges of the noise barriers, we use the results presented in [19].

First, we calculate the sound level and the sound pressure level (SPL) in each $j$-th octave band without the noise barrier:

$$
L_{A}=L_{A}^{e q}-\Delta L_{A}^{\text {dist }}-\Delta L_{A}^{\text {air }}-\Delta L_{A}^{\text {surf }}-\Delta L_{A}^{\frac{W}{T}}-\Delta L_{A}^{\text {green }}-\Delta L_{A}^{\alpha}, \mathrm{dBA}
$$




$$
\text { and } L_{j}=L_{A}+\Delta L_{T, j}, \mathrm{~dB} \text {, }
$$

where $\Delta L_{A}^{\text {dist }}$ - reduction of sound due to divergence of acoustic waves over distance; $\Delta L_{A}^{a i r}$ - losses during sound propagation in air from the sound source to the estimated point; $\Delta L_{A}^{\text {surf }}$ - absorption of sound by the territory surface;

$\Delta L_{A}^{\frac{W}{T}}$ - influence of the air turbulence and wind on the propagation of acoustic waves from the sound source to the estimated point;

$\Delta L_{A}^{\text {green }}$ - sound attenuation in the green belt;

$\Delta L_{A}^{\alpha}$ - correction related to the limited angle of visibility of a part of the road covered by the barrier from the estimated point.

$\Delta L_{T, j}$ - correction of the traffic noise spectrum shape in each $j$-th octave band. Correction values are either determined experimentally or taken from Table 1 [21].

Table 1. Traffic noise spectrum correction estimates

\begin{tabular}{|l|l|l|l|l|l|l|l|l|}
\hline $\mathrm{f}_{\text {avg. }, \mathrm{j}, \mathrm{Hz}}$ & 63 & 125 & 250 & 500 & 1000 & 2000 & 4000 & 8000 \\
\hline$\Delta L_{T, j}, \mathrm{~dB}$ & +13 & +7 & +2 & -3 & -7 & -10 & -16 & -22 \\
\hline
\end{tabular}

Second, we calculate the noise characteristic $L_{A}^{e q}$ of the traffic flow using empirical equation (19).

Using empirical formulas, we calculate noise reduction due to divergence of the sound waves over distance $\Delta L_{A}^{\text {dist }}$, the losses due to sound propagation in air $\Delta L_{A}^{\text {air }}$ and absorption of sound by the territory surface $\Delta L_{A}^{\text {surf }}$ using data from [20]:

$$
\Delta L_{A}^{\text {dist }}=10 \lg \left[\sqrt{\left(h_{1}-h_{2}\right)^{2}+c_{1}^{2}}+\left(\frac{s}{4}\right)^{2}\right]-\sqrt[3]{s}\left(\ln s-\frac{\sqrt{(s-3.8)^{2}}}{s}\right)-8,3, \mathrm{dBA},
$$

The error of the calculation using equation (52) relative to the initial data in the range $20 \leq c_{1}<1000 \mathrm{~m}$ is less than $0,5 \mathrm{dBA}$.

$$
\Delta L_{A}^{\text {air }}=0,005 \sqrt{\left(h_{1}-h_{2}\right)^{2}+c_{1}^{2}}, \mathrm{dBA} .
$$

For acoustically soft territory:

$$
\Delta L_{A}^{\text {surf }}=5 \ln \left(c_{2} / h_{2}\right)-3,45 h_{1}-8,09, \mathrm{dBA},
$$

here $c_{2}=l+D$, m. For acoustically rigid surface, $\Delta L_{A}^{\text {surf }}=0$.

The correction term $\Delta L_{A}^{B / T}$, that takes into account turbulence of air and the influence of wind on the propagation of sound [13], is given by:

$$
\Delta L_{A}^{B / T}=\frac{3}{1.6+10^{5} / \mathrm{c}_{1}^{2}}, \mathrm{dBA} .
$$

The attenuation of sound in the green belt, if present, is approximated [13] as follows:

$$
\Delta L_{A}^{\text {green }}=0,08 z, \mathrm{dBA},
$$


where $10 \leq z \leq 100 \mathrm{~m}$ is the width of the green belt. For regular green areas, their noise attenuation is neglected, i.e. $\Delta L_{A}^{\text {green }}=0$.

We calculate the correction term related to the limited angle of visibility of the part of the road shielded by the noise barrier from the estimated point:

$$
\Delta L_{A}^{\alpha}=10 \lg \left(\frac{\pi}{\alpha}\right), \mathrm{dBA},
$$

where $\alpha$ - the angle of visibility of the barrier, rad.

The study presented in [19] obtained equations for calculating acoustic efficiency of the noise barrier in each $j$-th octave band with diffraction of sound solely on the top edge of the noise barrier:

$$
\left\{\begin{array}{c}
\Delta L_{1, j}^{\mathrm{NB}}=10,8+4,8 \lg \left(\frac{F_{j}}{170}\right), \mathrm{dB} \quad \text { if } 17 \leq F_{j} \leq 68 \\
\Delta L_{1, j}^{\mathrm{NB}}=11,6+8,4 \lg \left(\frac{F_{j}}{170}\right), \mathrm{dB} \quad \text { if } 68<F_{j} \leq 14000 \\
\Delta L_{1, j}^{\mathrm{NB}}=28 \mathrm{~dB} \quad \text { if } F_{j}>14000
\end{array}\right.
$$

where $F_{j}=f_{j} \cdot \delta_{1}, \mathrm{~Hz} \cdot \mathrm{m}$;

$\delta_{1}=a_{1}+b_{1}-\sqrt{\left(h_{1}-h_{2}\right)^{2}+(\mathrm{l}+c)^{2}}, \mathrm{~m}$

$a_{1}=\sqrt{c^{2}+\left(h-h_{2}\right)^{2}}-$ distance from the acoustic center of the noise source to the upper edge of the noise barrier, $\mathrm{m}$;

$b_{1}=\sqrt{l^{2}+\left(h-h_{1}\right)^{2}}$ - distance from the noise barrier upper edge to the estimated point, $\mathrm{m}$; $c=a(s-0,5)+b_{s s w}+D, \mathrm{~m}$;

$b_{s s w}$ - separation strip width, m;

$f_{j}$ - center frequency of the $j$-th octave band, $\mathrm{Hz}$. dBA.

Relative to the initial data, the error of calculation using equation (58) is less than 0,5

Based on the data from [21], paper [19] obtained relations for evaluating the acoustic energy losses due to interaction of the sound waves with the territory surface with diffraction of sound the top edge of the noise barrier:

- for acoustically hard ground surface:

$$
\left\{\begin{array}{c}
\Delta L_{A}^{\text {surf }}=0 \text { if } 0,25 \leq \delta_{1} \leq 0,35 \mathrm{~m} ; \\
\Delta L_{A}^{\text {surf }}=-0,5 \mathrm{dBA} \text { if } 0,35<\delta_{1} \leq 0,65 \mathrm{~m} ; \\
\Delta L_{A}^{\text {surf }}=0,026142 \delta_{1}^{2}-0,01234 \delta_{1}^{3}-1,88582 \delta_{1}+0,13674, \mathrm{dBA} \\
\text { if } 0,65<\delta_{1} \leq 10 \mathrm{~m} ;
\end{array}\right.
$$

- for acoustically soft ground surface:

$$
\left\{\begin{array}{c}
\Delta L_{A}^{\text {surf }}=-0,5 \mathrm{dBA} \text { if } 0,2 \leq \delta_{1} \leq 1 \mathrm{~m} \\
\Delta L_{A}^{\text {surf }}=0 \text { if } 1<\delta_{1} \leq 1,45 \mathrm{~m} \\
\Delta L_{A}^{\text {surf }}=0,4 \mathrm{dBA} \text { if } 1,45<\delta_{1} \leq 1,75 \mathrm{~m} \\
\Delta L_{A}^{\text {surf }}=0,3 \mathrm{dBA} \text { if } 1,75<\delta_{1} \leq 2,95 \mathrm{~m} \\
\Delta L_{A}^{\text {surf }}=0 \text { if } 2,95<\delta_{1} \leq 10 \mathrm{~m}
\end{array}\right.
$$


Relative to initial data, the error of calculating $\Delta \mathrm{L}_{\mathrm{A}}^{\text {surf }}$ using formula (59) is less than 0,1 dBA in the range of $1,45<\delta 1 \leq 1,75 \mathrm{~m}$.

By plugging in the results of calculating $\Delta \mathrm{L}_{\mathrm{A}}^{\text {surf }}$ using formulas (59) and (60) in the equation (50) instead of equation (54) and passing over to the sound pressure level in each $j$ th octave band using equation (51), we calculate the spectrum of noise at the estimated point created by the sound radiation of the traffic flow diffracted over the noise barrier:

$$
L_{1, j}=L_{j}-\Delta L_{1, j}^{\mathrm{NB}}, \mathrm{dB} .
$$

Having summed the energy of octave band SPL levels corrected with the "A" characteristic, we calculate the corresponding sound level:

$$
L_{A, 1.1}=10 \lg \left(\sum_{j=1}^{8} 10^{0.1\left(L_{1, j}-\Delta L_{A, j}\right)}\right), \mathrm{dBA} .
$$

Then, we determine the contribution of the sound waves diffracted on the side edges of the noise barrier to the total noise in the estimated point created by the traffic flow. For this, we break down the part of the traffic flow limited by two rays passing though the edges of the barrier to the estimated point, Fig. 5. In the model, this part is represented as a line section A1 of the sound source equivalent line, broken down into finite elements with the length $\Delta 1$ [20]. The element length is a function of traffic speed and intensity; it is calculated using equations (28) - (31).

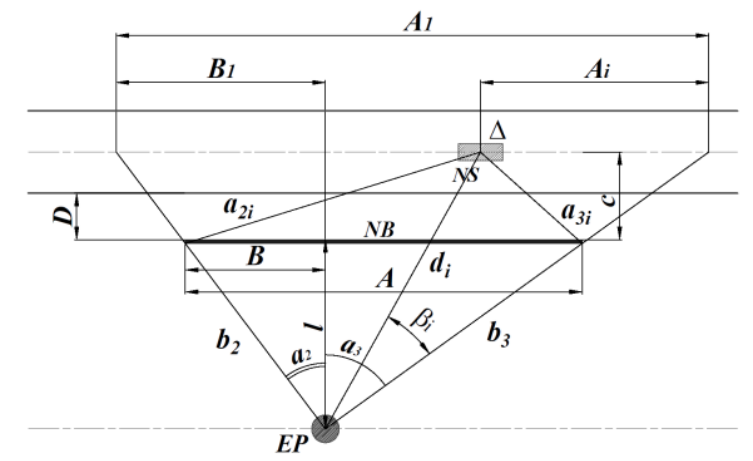

Fig. 5. Calculation diagram for determining noise barrier efficiency with diffraction on the side edges of the barrier of sound from the traffic flow

The difference of the lengths of the paths of the sound rays coming from the center of the $i$-th element to the estimated point directly and by going around the edges of the noise barrier is given by:

$$
\delta_{2,3, \mathrm{i}}=a_{2,3, i}+b_{2,3}-d_{i}, \mathrm{~m}
$$

where

$$
\begin{aligned}
& a_{2 i}=\sqrt{b_{2}^{2}+d_{i}^{2}-2 b_{2} \cdot d_{i} \cdot \cos \left(\alpha-\beta_{\mathrm{i}}\right)} \\
& a_{3 i}=\sqrt{b_{3}^{2}+d_{i}^{2}-2 b_{3} \cdot d_{i} \cdot \cos \left(\beta_{i}\right)}, \mathrm{m} \\
& b_{2,3}=\frac{1}{\cos \alpha_{2,3}}, \mathrm{~m}
\end{aligned}
$$




$$
\begin{aligned}
& d_{i}=\sqrt{(l+c)^{2}+\left(A_{1}-B_{1}-A_{i}\right)^{2}}, \mathrm{~m} ; \\
& \beta_{\mathrm{i}}=\alpha_{3}-\operatorname{arctg}\left(\frac{\left(A_{1}-B_{1}-A_{i}\right)}{l+c}\right), \mathrm{rad} ; \\
& B_{1}=B \cdot \frac{l+c}{l}, \mathrm{~m} ; \\
& \alpha=\alpha_{2}+\alpha_{3}, \mathrm{rad} ; \\
& A_{i}=\frac{\Delta(2 i-1)}{2}, \mathrm{~m} ; \\
& i=1,2 \ldots \mathrm{m} .
\end{aligned}
$$

The equivalent sound level in the estimated point generated by the $i$-th element of the traffic flow without taking into account noise attenuation during propagation:

$$
L_{A, \Delta}=L_{A}^{e q}-\Delta L_{A}^{\alpha}-10 \lg (m), \mathrm{dBA} .
$$

We determine individual the $j$-th octave sound pressure levels in the calculation level from the $i$-th element of the traffic flow with diffraction of sound on the side edges of the barrier to the left and right:

$$
\begin{aligned}
L_{2,3, i, j}= & L_{A, \Delta}-\Delta L_{2,3, i}^{\text {dist }}-\Delta L_{2,3, i, j}^{\text {air }}-\Delta L_{A, 2,3, i}^{\text {surf }}-\Delta L_{A, 2,3, i}^{W / T}-\Delta L_{A}^{\text {green }}-\Delta L_{2,3, i, j}^{\mathrm{NB}}+ \\
& +\Delta L_{T, j}, \mathrm{~dB}
\end{aligned}
$$

where $\Delta L_{A, 2,3, i}^{\text {surf }}, \Delta L_{A, 2,3, i}^{W / T}$ and $\Delta L_{A}^{\text {green }}$ are calculated using formulas (54), (55) and (56), accordingly;

$\Delta L_{T, j}$ - see Table 1

$$
\Delta L_{2,3, i}^{\text {dist }}=20 \lg \left(\frac{a_{2,3, i}+b_{2,3}}{7,5}\right), \mathrm{dB}
$$

$\Delta L_{2,3, i, j}^{a i r}$ are calculated using the method presented in [20] taking into account temperature $\mathrm{T}$ $[\mathrm{K}]$, relative humidity $\varphi[\%]$ and ambient pressure $\mathrm{p}_{\mathrm{a}}[\mathrm{kPa}]$ :

$$
\Delta L_{2,3, i, j}^{a i r}=\Delta \mathrm{w}_{\mathrm{j}}\left(a_{2,3, i}+b_{2,3}\right), \mathrm{dB},
$$

Based on the data presented in [19] derived equations for calculating the reduction of noise with diffraction on the side edges of the noise barrier:

$$
\left\{\begin{array}{c}
\Delta L_{2,3, i, j}^{\mathrm{NB}}=8+4,4 \lg \left(\frac{F_{2,3, i, j}}{17}\right), \mathrm{dB} \quad \text { if } 17 \leq F_{2,3, i, j} \leq 150 \\
\Delta L_{2,3, i, j}^{\mathrm{NB}}=1,9+11 \lg \left(\frac{F_{2,3, \mathrm{i}, j}}{17}\right), \mathrm{dB} \quad \text { if } 150<F_{2,3, i, j} \leq 14000 \\
\Delta L_{2,3, i, j}^{\mathrm{NB}}=34 \mathrm{~dB} \quad \text { if } F_{2,3, i, j}>14000 \\
F_{2,3, i, j}=f_{j} \cdot \delta_{2,3, \mathrm{i}}, \mathrm{Hz} \cdot \mathrm{m}
\end{array}\right.
$$

where $\delta_{2,3, \mathrm{i}}$ are calculated using equations (63)-(72). 
Relative to the initial data, the error of calculating $\Delta L_{2,3, i, j}^{\mathrm{NB}}$ using equation (77) is less than $0,3 \mathrm{~dB}$.

Having summed the energy SPL in the estimated point for all $\mathrm{m}$ elements of the traffic flow in each $j$-th octave band, we obtain spectra of the noise diffracted on the left and right edges of the noise barrier:

$$
L_{2,3, j}=10 \lg \left(\sum_{\mathrm{i}=1}^{\mathrm{m}} 10^{0.1 L_{2,3, i, j}}\right), \mathrm{dB}
$$

Or by the sound level:

$$
L_{A, 2,3}=10 \lg \left(\sum_{j=1}^{8} 10^{0.1\left(L_{2,3, j}-\Delta L_{A, j}\right)}\right), \mathrm{dBA} .
$$

Octave band noise spectrum and the level of noise diffracted on both side edges and the whole open perimeter of the noise barrier is given by:

$$
\begin{aligned}
& L_{\text {side }, j}=10 \lg \left(10^{0.1 L_{2, j}}+10^{0.1 L_{3, j}}\right), \mathrm{dB} ; \\
& L_{A, \text { side }}=10 \lg \left(10^{0.1 L_{A, 2}}+10^{0.1 L_{A, 3}}\right), \mathrm{dBA} \\
& L_{j}^{N B}=10 \lg \left(10^{0.1 L_{1, j}}+10^{0.1 L_{\text {side }, j}}\right), \mathrm{dB} ; \\
& L A 2=10 \lg \left(10^{0.1 L_{A, 1.1}}+10^{0.1 L_{A, \text { side }}}\right), \mathrm{dBA} .
\end{aligned}
$$

\section{Calculation of total noise level in the estimated point}

Using the formulas presented above, we determine the total noise level in the estimated point taking into account reduction of acoustic energy due to diffraction on the top and side edges of the noise barrier of the sound reflected from the road surface, from the noise barrier, the noise barrier on the opposite side of the road, and the parts of the traffic flow not shielded by the noise barrier.,

$$
L A=10 \lg \left[10^{0,1 L_{r e f l, \Sigma}}+10^{0,1 L A 1}+10^{0,1 L A 2}\right] .
$$

where $L_{r e f l, \Sigma}$ - the total level of sound reflected from the road surface, from the noise barrier, from the barrier on the opposite side of the road, if present, as well as from both barriers installed on both sides of the road;

$L A 1$ - noise level on the protected territory created by the parts of the traffic flow not shielded by the noise barrier;

$L A 2$ - noise level in the estimated point with diffraction of sound on the top and side edges of the noise barrier.

\section{Conclusion}

The proposed comprehensive acoustic calculation method enables designing noise barriers taking into account different factors. These factors include noise diffracted along the whole outer perimeter of the barrier, as well as the sound reflected from the opposite barrier, if present, and parts of the traffic flow unshielded by the noise barrier. During design process, the designer can identify where most of the noise is coming from, whether from the top of the screen or from its sides, including the noise from the parts of the traffic flow unshielded 
by the noise barrier to the left and to the right of the barrier, if the noise levels violate regulations. Thus, there is a possibility of rationally choosing geometric dimensions of the barrier by either increasing its height or increasing its length in either direction.

The design process determines the optimal geometric dimensions of the barrier. The proposed approach allows reducing design time of noise barriers and reduce noise barrier installation expenses.

\section{References}

1. N. D. Chaynov, V. A. Markov, A. A. Savastenko, Structural, Noise and Acoustic Characteristics Improvement of Transport Power Plants. In IOP Conference Series: Materials Science and Engineering, 327(2), 22-69 (2018)

2. A.I. Komkin, A.I. Bykov, Acoustical Physics, 62(3), 269-279 (2016)

3. A.I. Komkin, M.A. Mironov, A.I. Bykov, Acoustical Physics, 63(4), 385-392 (2017).

4. S. M. Sivachev, L. L. Myagkov, International Conference on Industrial Engineering, 109-117 (2019)

5. R. I. Rakhmatov, V. E. Krutolapov, V. N. Zuzov, Vibroengineering Procedia, 25, 135138 (2019)

6. Y. Kawai, K. Flujimoto, T. Itow, Noise propagation around a thin half plane. Acustica, 38, 313-323 (1978)

7. Y. Kawai, Sound attenuation by finite barriers, Proc. 11-th ICA, Paris, 1, 129-132 (1983)

8. T. Terai, J.S. a V., 69(1), 71-100 (1980)

9. U.J. Kurze, JASA, 55(3), $504-518$ (1974)

10. N. Kohshi, F. Katsuo, Boundary element analysis of the sound field around a finite barrier, J. Jap. Soc. Precis. Eng., 54, 1351 - 1356 (1988)

11. N.I. Ivanov, N.V. Tyurina, Proceedings of the International Scientific and Technical Conference, 9 - 36 (2006)

12. V.P. Bobrovskikh, N.I. Ivanov, N.V. Tyurina, On the coefficient of diffraction of acoustic screens, Workshop proceedings, Saint Petersburg: State Technological University, 141 - 147 (2007)

13. Guidelines for evaluating the required noise reduction near residential areas and determining the required acoustic efficiency of noises barriers taking into account sound absorption. Moscow: Ministry of Transport of the Russian Federation, 38 (2003)

14. V.V. Kuroedova, V.V. Tupov, Acoustics of the Environment, 95-102 (2016)

15. I.L. Schubin, I.E. Tsukernikov, N. Nikolov, A. Pisarski, Basics of design of road noise barriers, 208 (2015)

16. N.I. Ivanov, Engineering acoustics of transport machines: handbook, 365 (1992)

17. Road industrial methodical document 218.2.013-2011. Guidelines for protection of nearroad residential areas from traffic noise. - Moscow: FSUE "Informavtodor", 160 (2011).

18. T.I. Dudiev, V.V. Tupov, Acoustics of the Environment, 78-88 (2018)

19. V.V. Tupov, O.A. Chereshneva, Safety in the Industrial Sphere, 5, 17 - 24 (2014)

20. GOST 31295.2 - 2005 (ISO 9613 - 2:1996). Acoustics. Attenuation of sound during propagation outdoors. Part 2. General method of calculation. International standard. 2007.

21. G.L. Osipov, Noise protection in urban planning, 96 (1993) 